\title{
Comparison of ELG and UV Galaxies from the SBS Survey
}

\author{
M. V. Gyulzadian \\ BAO, Byurakan 378433, Armenia, E-mail:mgyulz@bao.sci.am
}

\begin{abstract}
.
In this paper we compare the results of spectrophotometric observations for more then 40 emission line galaxies (ELG) without UV-excess and 70 galaxies with UV-excess (UV-galaxies) from the Second Byurakan Survey (SBS) carried out with the scanner on the 6-m telescope of the Special Astrophysical Observatory. The comparison was aimed to answer the questions: are there any essential differences between these two types of galaxies? And what is the fraction of AGN among ELG and UV-galaxies? To answer this question we have investigated some physical parameters. Great care was taken in classifying the spectra of these galaxies as "HII region-like" or "AGN-like" using a new diagnostic method for emissionline galaxies (Rola et al.). We find that $14 \%$ of emission line galaxies (ELG) have Sy2 and LINER-like spectra. The transition or ambiguous objects among them comprise $20 \%$. The fraction of LINER-like and Sy2 objects among UV-galaxies is $21 \%$ while that of the transition or ambiguous objects in UV-galaxies is $28 \%$. From SBS galaxies, $18 \%$ are Sy2s and LINER-like galaxies.
\end{abstract}

\section{Introduction}

In 1978 the Byurakan Observatory marked the completion of the First (FBS) and the beginning of the Second Byurakan Sky Survey (SBS). The SBS is an objective prism survey, which was carried out with the Byurakan Observatory 1$\mathrm{m}$ Schmidt telescope in combination with a set of three low dispersion objective prisms $\left(1.5^{\circ}, 3^{\circ}, 46^{\circ}\right)$. The prisms' dispersions were respectively 1800,900 and $280 \AA / m m$ at $\mathrm{H} \gamma$. The observations were done on well-sensitized plates, which gave an opportunity to observe objects down to 19-20 magnitude. In the observed fields with an area of about 1000 square degrees $\left(\left(=8 \mathrm{~h}-17 \mathrm{~h},\left(=49^{\circ}-61^{\circ}\right)\right.\right.$, nearly 1300 nonstellar (galaxies) objects and 1700 star-like objects were discovered.

As in the case of the First Byurakan Survey (FBS, Markarian 1967) with the 1.5-degree prism, unwidened spectra were obtained and UV-excess objects were selected. Similarly to the FBS, the SBS classified objects as stellar ("s") or diffuse (" $\mathrm{d}$ ") type. In addition, from observations with the 3- and 4-degree prisms a large number of emission-line galaxies without UV-excess were identified. They are called emission-line galaxies (ELG) and also classified as stellar 
("s") or diffuse ("d") type, with "sd" and "ds" intermediate types without UVexcess numbers 1,2 ,or 3 (only s; sd; ds; d).

\section{Observation and Reduction}

Spectra (in the spectral range $3500 \AA-5800 \AA$ ) of galaxies discovered at the observatory were obtained at the Nasmyth focus of the SAO 6-m telescope with the SP-124 spectrograph with a dispersion of $100-200 \AA / m m$ (Stepanian et al. 1990). In this paper the results for more then 110 galaxies from the SBS are presented. The galaxy spectral observations were carried out during 1992 on the 6-m telescope of the Special Astrophysical Observatory using the 1024-channel photon counting scanner (Stepanian et al. 1992a, Stepanian et al. 1992b). The data were reduced with the AIDA system in BAO. From these galaxies some galaxies with UV-excess (UV-galaxies) and emission line galaxies (ELG) (without UV-excess) were chosen (Gyulzadian 1995a,b).

\section{Spectral Classification}

A good segregation between starburst and active galactic nuclei, i.e. Sy $2 \mathrm{~s}$ and LINERs, is obtained from diagnostic diagrams involving the $[O I I] \lambda 3727 \AA$ and $[O I I I] \lambda 5007 \AA$ relative intensities or the $[O I I] \lambda 3727 \AA$ and $H \beta$ equivalent widths. Furthermore, the color index of the continuum underlying $[O I I] \lambda 3727 \AA$ and $H \beta$ provides an additional separation parameter between the two types of emission line galaxies. We have classified the spectra of these galaxies as "HII region-like" or "AGN-like" using a diagnostic method for emission-line galaxies. The first $\mathrm{H}$ II region-like object among Mrk galaxies was Mrk 94 (Arp \& Khachikian 1974); forty objects were found subsequently by Sahakian \& Khachikian (1975). Future work will include the comparison with other H II - or AGN-like galaxies (e.g., Burenkov 1991). $66 \%$ of ELG, $36 \%$ of UV-galaxies and $54 \%$ of our total SBS sample are HII galaxies. We find that $14 \%$ of emission line galaxies (ELG) have Sy2 and LINER-like spectra. The transition or ambiguous objects among them comprise $20 \%$. The fraction of LINER-like objects and Sy2 among UV-galaxies is 21\%; the transition or ambiguous objects comprise $28 \%$. Among the whole sample of SBS galaxies $18 \%$ are Sy2 and LINER-like galaxies. In HII galaxies the emission-line spectrum is dominated by the emission originating in HII regions, where ultraviolet photons emitted by OB stars ionize the surrounding gas, while in the case of active galaxies, the gas-ionizing source is much harder and has the shape of a power law. Furthermore, star-forming regions are always associated with HII regions and notably with HII galaxies, while that is not necessarily the case for active galaxies. An active nucleus can disrupt the surrounding gas through its intense radiation field, X-ray heated wind, and possible radio jets (Begelman et al. 1983, Begelman et al. 1984).

The transition/ambiguous objects among SBS galaxies are located mainly with the H II galaxies in the new diagrams with little spillover into the AGN region. This could lead essentially to three possibilities: (1) These objects are in fact H II/AGN composites, with an AGN component weaker than the emission component produced from ionization by stellar sources; (2). They are H II 
galaxies with a peculiar hot stellar population; (3) these galaxies just have different properties in their ionized regions. It is difficult to determine what makes these objects different from $\mathrm{H}$ II galaxies.

We have done the SBS sample identification with IRAS objects. $30 \%$ of UV galaxies and $30 \%$ of ELG are IRAS objects.

Acknowledgments. I would like to thank J. Stepanian for the observational material.

\section{References}

Arp, H. \& Khachikian, E. Ye., 1974, Astrofiz., 11, 207, 1975.

Begelman, Blandford, \& Rees, 1984, Rev.Mod.Phys. 6255.

Begelman, McKee, \& Shields, 1983,APJ.,271 70.

Burenkov, A. N., 1991, Preprint of the SAO, No. 67.

Gyulzadian, M., 1995a, Astrofiz., 32501.

Gyulzadian, M., 1995b, IAU 171386.

Markarian, B.,1967, Astrofiz., 355.

Rola, C.S., Terlevich, E., and Terlevich, R.,1997, MNRAS, 289, 419.

Sahakian, K. A. \& Khachikian, E. Ye., 1975, Astrofiz., 11, 207.

Stepanian, J. et al., 1990, Astrofiz., 389.

Stepanian, J., Lipovetsky, V., Erastova, L. Shapavalova, A., Gyulzadian, M., 1992a, Astrofiz. Issled., 3515.

Stepanian, J., Lipovetsky, V., Erastova, L., Gyulzadian, M. Izotov, Yu., Guseva, N., 1992b, Astrofiz. Issled., 3532. 